\title{
Adamina, Marco; Kübler, Markus; Kalcsics, Katharina; Bietenhard, Sophia \& Engeli, Eva (Hrsg.) (2018): ,Wie ich mir das denke und vorstelle ..." Vorstellungen von Schülerinnen und Schülern zu Lerngegenständen des Sachunterrichts und des Fachbereichs Natur, Mensch, Gesellschaft
}

\section{Bad Heilbrunn/Obb.: Klinkhardt, 372 S., € 19,90}

\author{
Sandra Tänzer • Andreas Raith \\ Online publiziert: 23. Januar 2020 \\ (C) Der/die Autor(en) 2020
}

Die letzte, achte Unterrichtsstunde zum Thema Schwimmen und Sinken läuft. [...] Da meldet sich Josef [...] ziemlich aufgeregt: ,Jetzt weiß ich, jetzt weiß ich, das ist gar nicht der Lack, der das macht, das ist das Wasser, das Wasser drückt das hoch!“. Ich werde stutzig und frage nach, was er mit dem Lack meine. Er erzählt, dass er die ganze Zeit [...] gedacht habe, das Schiff schwimmt, weil es lackiert ist, und jetzt habe er verstanden, dass es nicht am Lack liegt. Ich frage nach, wieso er gedacht habe, es liege am Lack. Seine Antwort: Weil er im Kindergarten einmal Papierschiffe gefaltet habe. Und diese wurden dann außen mit Wachsstiften bemalt, ganz dick, damit das Schiffchen nicht untergeht. Und es sei ja auch nicht untergegangen. Und die Wachsfarbe sei doch genauso wie der Lack beim Schiff (S. 35).

Mit diesem anschaulichen Beispiel beginnt der Beitrag von Kornelia Möller in dem Buch „,Wie ich mir das denke und vorstelle ... '. Vorstellungen von Schülerinnen und Schülern zu Lerngegenständen des Sachunterrichts und des Fachbereichs Natur, Mensch, Gesellschaft", von Marco Adamina, Markus Kübler, Katharina Kalcsics, Sophia Bietenhard und Eva Engeli 2018 im Klinkhardt-Verlag herausgegeben. Es ist einer von 18 Beiträgen dieses 372 Seiten umfassenden Werkes, an dem $26 \mathrm{Au}$ tor*innen aus Deutschland und der Schweiz mitwirkten.

Das Anliegen des Werkes ist es, mit dem hier verdichteten Überblick über ,domänenspezifische Vorstellungen der Kinder über die Welt“ (S. 9) einem in der Tat überfälligen Desiderat entgegenzutreten, gelten doch die Wahrnehmung und Deu-

Prof. Dr. S. Tänzer $(\varangle) \cdot$ Dr. A. Raith

Fachbereich Sachunterricht/Schulgarten, Universität Erfurt, Erfurt, Deutschland

E-Mail: sandra.taenzer@uni-erfurt.de

Dr. A. Raith

E-Mail: andreas.raith@uni-erfurt.de 
tung von und ein angemessener Umgang mit Schülervorstellungen als ein entscheidender Prädiktor für verständnisfördernden, lernwirksamen Unterricht.

Der 1. Teil des Buches ist grundlagentheoretisch ausgerichtet und eine hervorragende Quelle für Studierende, Lehrende und Forschende, um theoretische Zusammenhänge über das Thema dicht und prägnant zu erfassen.

Ilonca Hardy und Nicole Meschede erörtern lern- und entwicklungspsychologische Grundlagen, Kornelia Möller setzt sich mit der Bedeutung von Schülervorstellungen für das Lernen im Sachunterricht und im Fachbereich Natur Mensch Gesellschaft (kurz: NMG) auseinander, Andreas Hartinger und Lydia Murmann fragen nach Methoden ihrer Erfassung und geben Einblicke in Anforderungen der Analyse und Diagnose.

Leser*innen erwarten neben begrifflichen und semantischen Klärungen Einblicke in Erhebungsinstrumente zur Erfassung von Schüleräußerungen und deren Deutung im Hinblick auf die zum Ausdruck kommenden Schülervorstellungen sowie historische und gegenwärtige Ansätze zu deren Berücksichtigung und dem Umgang mit ihnen.

Alle Beiträge tragen zur Klärung dieses komplexen Konstrukts „,Schülervorstellung“ bei, wobei gleichsam Kontraste in deren Deutung und Bewertung auffallen: Werden sie als im Vergleich zu wissenschaftlichen Vorstellungen unvollständige oder fehlerhafte Vorstellungen (S. 25, S. 139) gedeutet oder als kontextbedingte plausible und persönlich sinnhafte Überlegungen innerhalb eines früheren Lernprozesses, wie das Eingangsbeispiel des kleinen Josef oder auch ein wunderbar anschauliches Beispiel über Wind, Windräder, Propeller und Föhne im Beitrag von Andreas Hartinger und Lydia Murmann zeigt?

Die Beiträge des 2. Teils des Buches setzen sich mit bereichsspezifischen Schülervorstellungen zu Themen des Sachunterrichts und des Fachbereichs NMG auseinander. Alle 13 Beiträge gehen dabei weit über eine Darstellung des Forschungsstandes hinaus, indem sie - wenn auch in Art und Ausprägung sowie innerer Kohärenz unterschiedlich - zudem

- in Ziele und Anliegen einzelner Domänen wie das Historische, das Politische oder auch das Technische einführen,

- systematisch wissenschaftliche Vorstellungen zu den ausgewählten Themenfeldern darstellen und diese fachliche Klärung mitunter sehr geschickt mit empirischen Befunden zu Schülervorstellungen sowie didaktischen Schlussfolgerungen verknüpfen,

- Einflussfaktoren auf Prozesse der Vorstellungsbildung hervorheben sowie die Rolle der Lehrperson reflektieren und

- aus Forschungsperspektive teilweise die Auswahl der Studien begründen und die referierten Studien nicht nur darstellen, sondern kritisch kommentieren und auf Forschungslücken hinweisen.

Alle Aufsätze im Einzelnen zu besprechen, würde diese Rezension sprengen, so dass nachfolgend nur ein grober Überblick gegeben wird.

Themenfelder des naturwissenschaftlichen und geographischen Lehrens und Lernens werden behandelt in den Beiträgen von Marcus Schrenk und Petra Baisch (Lebewesen - Tiere, Pflanzen, Ökologie, Lebensräume), Jan Christopf Hadenfeld, 
Irene Neumann, Knut Neumann und Mirjam Steffensky (Stoffe, Energie, Bewegung), Florian Böschl, Thomas Ottlinger und Kim Lange-Schubert (Sinne, Optik, Akustik) und Jan Christoph Schubert (naturwissenschaftlich-geographische Phänomene wie Jahreszeiten, Wasserkreislauf oder Wetterphänomene). Die geographische Perspektive ist zudem mit Bezug zu den Themenfeldern Raum(wahrnehmung), räumliche Orientierung, Erde, ferne Räume und Lebensweisen von Menschen in verschiedenartigen Räumen in zwei Beiträgen von Marco Adamina vertreten. Forschungen zu kindlichen Präkonzepten über Technik, wie Brücken oder die Funktionsweise von Staubsaugern, Fahrrädern und Handbohrmaschinen, und daraus folgende didaktische Implikationen stellt der Beitrag von Kornelia Möller und Hans-Peter Wyssen vor.

Anforderungen an historisches Lehren und Lernen zeigt Markus Kübler vor dem Hintergrund von Schülervorstellungen über Zeit, Dauer und Wandel auf.

Aus sozialwissenschaftlicher Perspektive setzen sich Michel Dängeli und Katharina Kalcsics mit Vorstellungen von Kindern über die politische Ordnung, Öffentlichkeit und Meinungsvielfalt sowie das Gemeinwohl auseinander. Anne-Marie Gafner Knopf und Meike Wulfmeyer reflektieren Präkonzepte von Kindern zu wirtschaftlichen Phänomenen (Arbeitslosigkeit, Berufe, Geld, Kaufen und Konsum), während im Fokus von Sophia Bietenhard, Anne-Marie Gafner Knopf und Barbara Jaun-Holderegger Aspekte der Befindlichkeit von Kindern wie Glück, Gesundheit, Krankheit und der menschliche Körper stehen.

Mit den Beiträgen von Sophia Bietenhard und Dominik Helbling rücken philosophische und religionskundliche Themenfelder des Schweizer NMG-Unterrichts in den Mittelpunkt der Betrachtung. Sophia Bietenhard thematisiert Schülervorstellungen über ,Lebenssinn, Lebensgestaltung, existentielle() Erfahrungen sowie philosophische() und ethische() Grundbegriffe()“ (S. 270) wie Angst, Tod und Trauer, Gerechtigkeit oder Glück. Dominik Helbling erläutert kindliche Deutungs- und Erklärungsmuster religiöser Phänomene wie religiöse Symbole, Texte, Praxen oder religiöse Vielfalt. Das Spannende dieser beiden Beiträge ist nicht nur ihre Linienführung im Sinne der oben genannten inhaltlichen Aspekte aller Beiträge. Sie fordern zugleich zu vergleichenden Reflexionen über curriculare Strukturen der Fächer Sachunterricht und NMG heraus.

Der Blick auf perspektivenvernetzende Themenbereiche wird im vorliegenden Buch auf Aspekte der Gesundheit und Nachhaltigkeit beschränkt.

Den Abschluss des Buches (3. Teil) bildet ein Aufsatz von Marco Adamina über Interessen von Schüler*innen, da diese in enger Wechselbeziehung zum Vorwissen stehen. Aus Sicht der Forschung wäre es sicher auch empfehlenswert gewesen, analytisch die 13 Beiträge des Mittelteils nach trennenden und verbindenden Aspekten der Schülervorstellungsforschung über die Domänen des Sach- und NMG-Unterrichts hinweg zu untersuchen, methodologische und methodische Reflexionen anzuschließen und damit einen Beitrag auf einer metatheoretischen Ebene zu diesem Forschungsfeld zu leisten.

Was ist die große Leistung dieses Buches? Aus Forschungsperspektive führt es den theoretischen Diskurs in diesem wichtigen Forschungsfeld zusammen, kann Potentiale und Grenzen sowie Forschungslücken prägnanter benennen. Aus der Perspektive des Unterrichts erhalten Lehrkräfte mit dieser außerordentlich systematischen und komplexen Darstellung theoretischer Zusammenhänge sowie älterer wie 
auch aktueller empirischer Befunde über Schülervorstellungen im didaktischen Kontext eine umfassende Grundlage, um ihr fachliches und fachdidaktisches Wissen zu erweitern.

Gleichsam ersetzt das Buch nicht die Erfassung der heterogenen Schülervorstellungen der eigenen Klasse, im Gegenteil: Man muss es, auch wenn das auf Textebene nicht in jedem Beitrag deutlich wird, als Aufforderung zur Wahrnehmung und dem Verstehen individueller kindlicher Denk- und Erklärungsmuster - geprägt von u.a. sozioökonomischen, kulturellen Gegebenheiten ihrer Lebenswelt - lesen und wird aus der Innenperspektive des eigenen Unterrichts auf vielgestaltige Vorstellungen der Kinder treffen, wie auch Marco Adamina mit Bezug zu globalen Fragen und der Zukunft unserer Erde unterstreicht: „Während die einen Lernenden noch gar keine Bezugspunkte und Stichworte zum Thema aufführen, kennen andere bereits verschiedene Aspekte des Klimawandels und stellen diese auch in einen Zusammenhang" (S. 219).

Das vorliegende Buch orientiert sich lerntheoretisch konsequent an einem (pädagogisch-psychologisch fundierten) konstruktivistischen Verständnis des Lernens. Nur vereinzelt (wie beispielsweise im Beitrag von Anne-Marie Gafner Knopf und Meike Wulfmeyer) werden Bezüge zu für sinnkonstituierenden und sinnstiftenden Unterricht elementaren bildungstheoretischen Ansätzen hergestellt. Gänzlich offen bleibt leider eine Bezugnahme zur Rolle und Bedeutung von Vorwissen, Vorstellungen und Vorerfahrungen von Kindern in alternativen - eher philosophischen Traditionen folgenden - lerntheoretischen Ansätzen, insbesondere der phänomenologischen Lerntheorie, die Lernen als Erfahrung und nicht als Conceptual Change begreift und lebensweltlichen, vor- und außerschulischen Erfahrungen für Erkenntnisbildungsprozesse eine große Bedeutung zuweist.

Nichtsdestotrotz soll mit dieser einschränkenden Aussage der Gewinn des Buches nicht geschmälert werden und es ist ihm zu wünschen, dass es viele Leser*innen findet.

Funding Open Access funding provided by Projekt DEAL.

Open Access Dieser Artikel wird unter der Creative Commons Namensnennung 4.0 International Lizenz veröffentlicht, welche die Nutzung, Vervielfältigung, Bearbeitung, Verbreitung und Wiedergabe in jeglichem Medium und Format erlaubt, sofern Sie den/die ursprünglichen Autor(en) und die Quelle ordnungsgemäß nennen, einen Link zur Creative Commons Lizenz beifügen und angeben, ob Änderungen vorgenommen wurden.

Die in diesem Artikel enthaltenen Bilder und sonstiges Drittmaterial unterliegen ebenfalls der genannten Creative Commons Lizenz, sofern sich aus der Abbildungslegende nichts anderes ergibt. Sofern das betreffende Material nicht unter der genannten Creative Commons Lizenz steht und die betreffende Handlung nicht nach gesetzlichen Vorschriften erlaubt ist, ist für die oben aufgeführten Weiterverwendungen des Materials die Einwilligung des jeweiligen Rechteinhabers einzuholen.

Weitere Details zur Lizenz entnehmen Sie bitte der Lizenzinformation auf http://creativecommons.org/ licenses/by/4.0/deed.de. 